\title{
Ixodes randrianasoloi sp. n. (Ixodoidea, Ixodidae) parasite de Rattus rattus (Rodentia) à Madagascar
}

par Gerrit UILENBERG ${ }^{2}$ et Harry HOOGSTRAAL ${ }^{3}$

\begin{abstract}
Résumé
Ixodes randrianasoloi sp. $\mathrm{n}$. est décrite d'après une femelle récoltée à Madagascar sur Rattus rattus. L'espèce se distingue nettement des six autres Ixodes connues dans le pays, en particulier par la forme des auriculae.
\end{abstract}

\section{Summary}

Ixodes randrianasoloi sp. $\mathrm{n}$. is described from a female collected in Madagascar on Rattus rattus. This species is easily distinguished from the six other Ixodes species known in the country, particularly by the form of the auriculae.

\section{IXODES RANDRIANASOLOI SP. N.}

Holotype : Femelle ex Rattus rattus (Rodentia), Massif du Tsaratanana, altitude $2.090 \mathrm{~m}$, Madagascar, 7 novembre 1966, récoltée par G. Randrianasolo. Holotype déposé dans la collection du Rocky Mountain Laboratory, Hamilton, Montana (RML 49, 290).

(1) From Research Project MR005.09-0028, Bureau of Medecine and Surgery, Department of the Navy, Washington, D.C. The opinions and assertions contained herein are the private ones of the authors and are not to be construed as official or as reflecting the views of the Department of the Navy or of the naval service at large. The illustrations in this report were prepared under the auspices of Agreement 352505 between the National Institutes of Health and NAMRU-3.

(2) Institut d'Elevag e et de Médecine Vétérinaire des Pays tropicaux. B.P. 39, Bouar (République Centrafricaine).

(3) Department of Medical Zoology, United States Naval Medical Research Unit Number Three, Le Caire, R.A.U. 


\section{DESCRIPTION.}

Femelle (seul stade connu; spécimen gorgé). Figs. 1 à 10.

La seule femelle récoltée et gorgée. La longueur totale $\times$ la largeur est de 7,0 $\times$ $4,5 \mathrm{~mm}$. La couleur du corps et de l'écusson est marron foncé, l'écusson étant plus foncé que l'alloscutum ; les pattes et le capitulum sont marron clair. (La tique a été conservée dans de l'alcool).

Le corps s'élargit vers l'arrière, pour se rétrécir à partir du niveau du tiers postérieur, et les bords latéraux s'unissent sur un bord postérieur droit.

Capitulum. La largeur de la basis capituli (mesurée en aspect dorsal) est de 0,49 mm, la longueur (entre la naissance de l'hypostome et le bord dorsal postérieur, dans la ligne médiane) de 0,28 à $0,29 \mathrm{~mm}$. La longueur totale du capitulum est de $0,74 \mathrm{~mm}$ (entre la pointe des palpes et le bord dorsal postérieur). Le bord dorsal postérieur est convexe, les bords postéro-latéraux sont pratiquement droits. Cornua courts, triangulaires, plus larges que longs, à extrémité arrondie. Areae porosae ovales, comme dessinées. Le bord ventral postérieur est convexe. Les auriculae forment des extensions aplaties, en rectangles allongés, dirigées postérolatéralement ; elles sont dépourvues d'éperon (fig. 4).

Les palpes sont relativement trapus; la longueur des segments II et III combinés est de $0,52 \mathrm{~mm}$, la plus grande largeur en aspect dorsal de $0,17 \mathrm{~mm}$. Le segment II constitue un peu plus de la moitié de la longueur combinée, environ $60 \%$. (Mensurations sur le bord latéral). Les bords latéraux des palpes sont légèrement concaves, les bords médiaux sont très convexes. Le bord médial de la moitié apicale du segment II est relevé en crête.

L'hypostome, en aspect dorsal, a une longueur de $0,44 \mathrm{~mm}$. La dentition est de $2 / 2$, mais à l'apex des petites dents couvrent, de façon irrégulière, toute la surface ventrale (L'hypostome est légèrement endommagé au milieu). L'extrémité des palpes dépasse légèrement celle de l'hypostome.

Scutum. Longueur $\times$ largeur $=1,17 \times 0,89 \mathrm{~mm}$ (les scapulae sont comprises dans la longueur). Rapport longueur $\times$ largeur $=1,31$. La plus grande largeur se trouve un peu en arrière de la mi-longueur.

Les bords antérolatéraux sont d'abord convexes, ensuite droits et divergents ; les bords postérolatéraux sont légèrement convexes, convergent et se réunissent en un arc. Les carinae latérales atteignent les bords postérolatéraux. Les fosses cervicales sont peu profondes, les sillons cervicaux sont peu marqués, mais atteignent les bords postérolatéraux (juste visibles en lumière tangente). L'écusson est glabre. Les ponctuations sont de taille moyenne, assez régulièrement distribuées, moins nombreuses sur la partie antérieure de l'écusson (Fig. 1).

Alloscutum. Soies courtes, peu nombreuses.

Pattes. Syncoxes sur coxae I, II et III. Eperon intérieur de la coxa I court, pointu, éperon extérieur à peine marqué. Coxae II et III sans éperon, coxa IV avec un éperon extérieur, court, pointu. Trochanters sans éperons. Tarses se rétrécissant assez brus- 

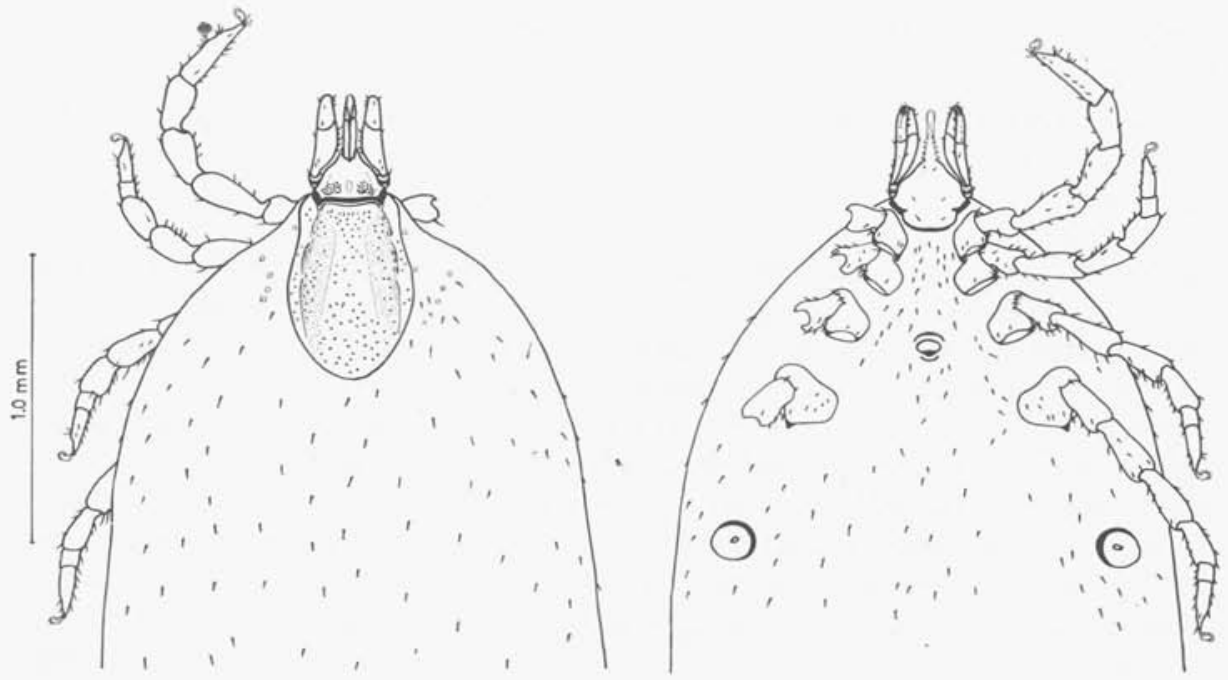

1

2

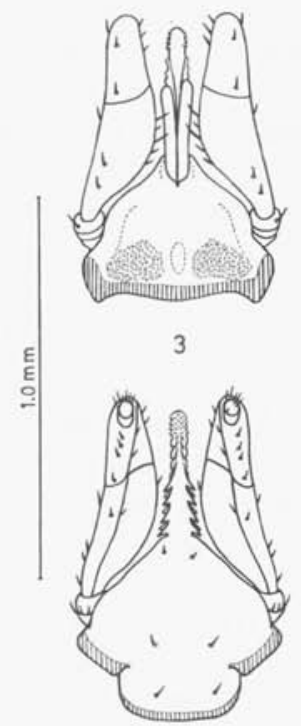

4

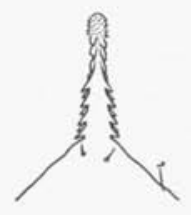

5

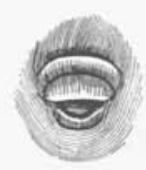

6

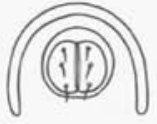

7
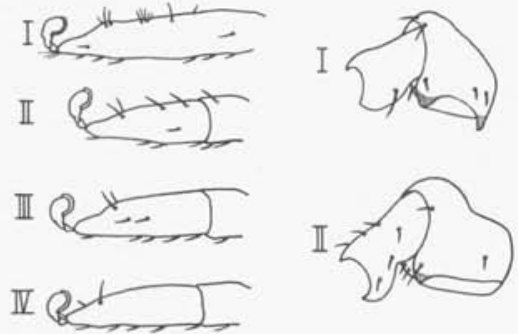

10
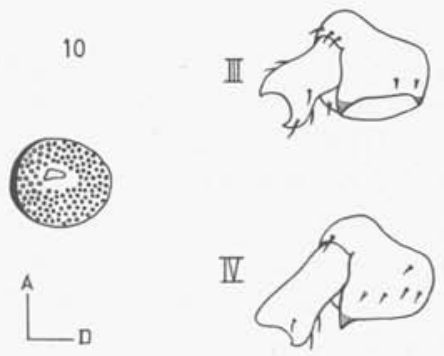

8

quement ; pas de bosse devant l'organe de Haller. Les pulvilli remplissent entièrement les crochets. Les soies sur les pattes sont courtes et peu nombreuses.

Gonopore. Au niveau du bord postérieur des coxae III (Fig. 6).

Stigmates. Dimensions de $0,27 \times 0,22$ à $0,23 \mathrm{~mm}$. La forme est allongée, avec l'axe long transversal par rapport à l'axe long de la tique; le bord postérieur est plus 
convexe que le bord antérieur. La macula est légèrement antérieure et ventrale au centre.

Sillon anal. Il forme un arc devant l'anus et les bords latéraux sont parallèles.

\section{DISCUSSION.}

1. randrianasoloi se distingue nettement de toutes les autres espèces de ce genre décrites de Madagascar. La forme des auriculae et l'absence d'éperons sur celles-ci l'en séparent immédiatement. L'absence d'éperons sur les coxae II et III, et la présence de syncoxes sur les coxae I, II et III la séparent en plus d'I. lunatus Neumann, d'I. lemuris Arthur et d'l. domerguei Uilenberg et Hoogstraal. Les autres espèces connues dans le pays jusqu'ici, I. colasbelcouri Arthur, I. albignaci Uilenberg et Hoogstraal, et $I$. nesomys Uilenberg et Hoogstraal, possèdent également des syncoxes sur les trois premières coxae et n'ont pas non plus d'éperon sur les coxae II et III. Quelques différences avec ces trois espèces, outre la forme des auriculae et l'absence d'éperon rétrograde chez I. randrianasoloi : les palpes de cette dernière sont plus trapus que ceux des autres espèces; les dimensions d' $I$. colasbelcouri et d' $I$. albignaci sont plus faibles que celles d'I. randrianasoloi; les éperons des coxae I et IV sont beaucoup plus longs sur I. colasbelcouri que sur 1 . randrianasoloi et les trochanters de la première possèdent des éperons ; l'écusson d'I. randrianasoloi est glabre, à l'opposé de celui des trois autres espèces ; les tarses d'I. randrianasoloi se rétrécissent assez brusquement, à l'envers de ceux des autres espèces ; les soies d'I. albignaci sont beaucoup plus nombreuses et longues que sur I. randrianasoloi; les ponctuations de l'écusson d'I. randrianasoloi sont beaucoup plus nombreuses que sur 1 . nesomys.

Il serait intéressant de savoir quel est l'hôte naturel de cette tique, Rattus rattus étant un animal introduit dans le pays.

\section{Bibliographie}

ARThUR (D. R.), 1957. - Re-description of lxodes lunatus Neumann, 1907 and of a new species confused therewith, from Madagascar. J. Parasit., 43: 474-483.

ARTHUR (D. R.), 1957. - The Ixodes schillingsi group : ticks of Africa and Madagascar, parasitic on primates, with descriptions of two new species (Ixodoidea, Ixodidae). Parasitology, $47: 544-559$.

Neumann (L. G.), 1907. - Note XI. Quatre espèces nouvelles d'Ixodidés. Notes from the Leyden Museum, 29: 88-100.

Uilenberg (G.) et Hoogstraal (H.), 1965. - Ixodes domerguei n. sp. (Ixodoidea: Ixodidae), the Madagascar bird ixodid. J. Med. Ent., 2: 258-260.

Uilenberg (G.) et Hoogstrat (H.), 1968. - Ixodes albignaci sp. n. (Ixodoidea, Ixodidae), parasite d'Insectivora et de Rodentia à Madagascar. Ann. Parasit. hum. comp., $43: 605-610$.

Uilenberg (G.) et Hoogstranl (H.), 1969. - Ixodes nesomys sp. n. (Ixodoidea, Ixodidae), parasite d'un rongeur malgache. Ann. Parasit. hum. comp., 44: 97-100. 